\title{
Implant Related MRONJ - A Case of Partial Mandibulectomy in the Medically Compromised Elderly Patient
}

\author{
Chan-Ho Jung, DDS ${ }^{1}$, Kwan-Soo Park, DDS, MSD, PhD²* \\ ${ }^{1}$ Resident, Department of Oral and Maxillofacial Surgery, Inje University Sanggye-Paik Hospital, College of \\ Medicine, Inje University, Seoul, Korea \\ ${ }^{2}$ Associate Professor, Department of Oral and Maxillofacial Surgery, Inje University Sanggye-Paik Hospital, \\ College of Medicine, Inje University, Seoul, Korea \\ *Corresponding author: Kwan-Soo Park, Department of Oral and Maxillofacial Surgery, Inje University \\ Sanggye-Paik Hospital, 1342, Dongil-ro, Nowon-gu, Seoul 01757, Korea. \\ Tel: +82-2-950-1161. Fax: +82-2-950-1167. E-mail: OMS_kspark@paik.ac.kr
}

\section{OPEN ACCESS}

pISSN 1229-5418

eISSN 2671-6623

Implantology 2020; 24(4): 183-192

https://doi.org/10.32542/implantology.202016

Received: July 29, 2020

Revised: September 28, 2020

Accepted: October 12, 2020

ORCID

Chan-Ho Jung

https://orcid.org/0000-0002-3967-5238

Kwan-Soo Park

https://orcid.org/0000-0002-0254-279X

Copyright $\odot$ 2020. The Korean Academy of Oral \& Maxillofacial Implantology

(c) (7) (3) $\begin{aligned} & \text { This is an Open Access article } \\ & \text { distributed under the terms of the }\end{aligned}$ distributed under the terms of the Non-Commercial License (http://creativecommons. org/licenses/by-nc/4.0/) which permits unrestricted non-commercial use, distribution, and reproduction in any medium, provided the original work is properly cited.

\section{Abstract}

The aim of this case report is to present the possibility of development of osteonecrosis around implant under load in a patient taking antiresorptive agent. A 78-year-old female complained of the mobility of implants and the swelling on left submandibular area. The patient was taking alendronate for the treatment of osteoporosis during 28 months. The implants were placed and functioned long before the patient received alendronate. Pus discharge and gingival swelling were observed on peri-implant area. Alveolar bone loss was seen on the radiograph. Curettage was performed and the antiresorptive agent was discontinued. Five months later, the patient revisited with swelling and new fistula formation on left submandibular area. On the radiograph, sequestrae and fracture line were seen around peri-implant area. Supportive care was performed for general anesthesia surgery. Partial mandibulectomy was performed, the result of the surgery was good and the reconstruction metal plate is maintained well.

Keywords: Dental implant, Implantitis, Medication-related osteonecrosis of the jaw, Partial mandibulectomy

\section{I . Introduction}

Bisphosphonates reduce osteoclast-mediated bone resorption in multiple myeloma, breast cancer and other solid carcinomas. ${ }^{1,2}$ Bisphosphonates are known to reduce osteoclast activity through several mechanisms such as inhibition of osteoclast development and increase of osteoclast apoptosis, then reduce bone resorption, hypercalcemia in some malignancies and osteolysis in bone metastases. However, normal osteoclasis is necessary for bone turnover and remodeling, and bisphosphonates affect the osteoclasis and induce avascular state in the jaws. In 2003, Marx reported that bisphosphonates may induce 
avascular necrosis of the jaws after oral and maxillofacial surgery including tooth extraction. ${ }^{3}$ Patients may be considered having bisphosphonates-related osteonecrosis of the jaws (BRONJ) if all of the following three characteristics are present: 1 . Current or previous treatment with bisphosphonates; 2 . Exposed, necrotic bone in the maxillofacial region that has persisted for more than 8 weeks; 3 . No history of radiation therapy to the jaws. ${ }^{4}$ In 2014, the American Association of Oral and Maxillofacial surgeons (AAOMS) changed the term BRONJ to medication-related osteonecrosis of the jaw (MRONJ). ${ }^{5}$ It is a concept that includes the osteonecrosis associated with not only bisphosphonates, but also other antiresorptive agents such as denosumab and antiangiogenic agents such as sunitinib, bevacizumab.

As described above, there is the possibility of development of MRONJ after not only tooth extraction but also implant placement, and there is concern about the failure of the implants. ${ }^{6}$ In general, it is known that dento-alveolar surgery is not strictly contraindicated in patients who orally take bisphosphonates, but it is suggested that dento-alveolar surgery should be carefully determined in oncology patients with intravenous antiresorptive or antiangiogenic therapies. According to the Korean Association of Oral and Maxillofacial Surgeons (KAOMS), the gudelines for "Dental management of patients receiving antiresorptive or antiangiogenic agents" indicate that dental treatment plans are not necessary to change in patients with duration of oral bisphosphonates 4 years or less and no specific clinical risk factors. But, in spite of the low possibility of development of MRONJ, it is recommended that surgery consent be taken including information about the possibility of osteonecrosis related to medication.

When placing implants in patients receiving antiresorptive or antiangiogenic therapies, clinicians pay great attention to the treatment plans with the possibility of development of MRONJ in mind. However, recently, it has been reported that MRONJ may develop around successfully-osseointegrated implants placed before antiresorptive or antiangiogenic therapies. It suggests that antiresorptive and antiangiogenic agents may affect on successfully-osseointegrated implants subjected to functional loading. Therefore, clinicians should be aware that implant-related MRONJ may occur not only after implant placement surgery but also around implants that are already present. ${ }^{7}$

With literature review, our case report documents the case of a elder patient with MRONJ who medically compromised, and the good treatment results with careful supportive care.

\section{Case Report}

A 78-year-old female visited authors' clinic with chief complaint of the mobility of implants located in the mandibular left posterior area (\#34, 35, 36 implant area) and the swelling on left submandibular 
area. The patient was taking an antihypertensive agent to control the hypertension, and medications for the improvement of cognitive function and the control of abnormal behaviors. Also, the patient had history of hepatitis $\mathrm{C}$, but was completely cured. For the treatment of osteoporosis, alendronate was administered $70 \mathrm{mg}$ once a week for 28 months. On clinical examination, there were mild swelling on left submandibular area, and mild mobility (mobility degree "1") of \#34, 35 implant fixtures which was restored with 4 units fixed prosthesis, moderate mobility (mobility degree "2") of \#36 implant fixture, peri-implant gingival swelling, and intrasulcus pus discharge. Opposite teeth was natural dentition and had mild periodontits. Panoramic and periapical radiographs were taken, but a clear image of the region of interest could not be obtained due to poor posture due to the patient's general conditions and uncooperative behavior (Fig. 1). On the periapical view, \#36 implant fixture and the prosthesis were not connected, and mild alveolar bone loss around \#36 implant was seen (Fig. 2). On these clinical examination, the impression were peri-implantitis and cellulitis, but the possibility of development of MRONJ was not ruled out. On the day of visit, peri-implant curettage was performed and antibiotics

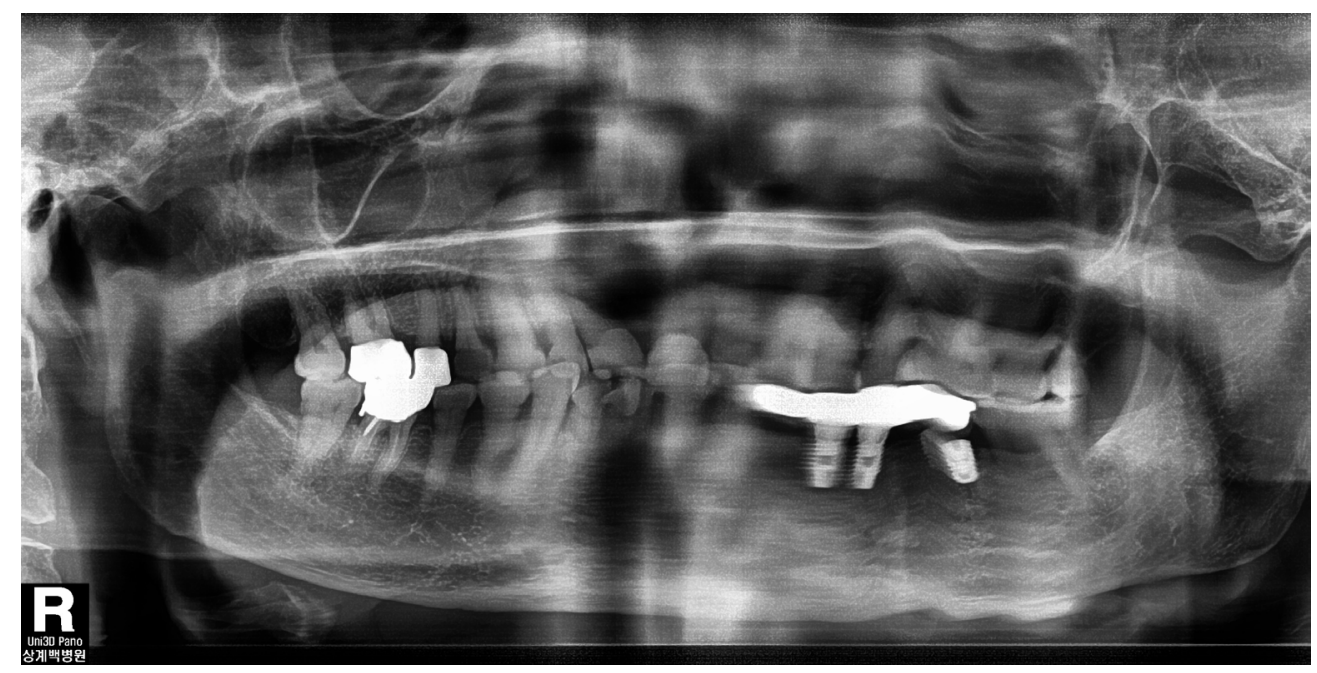

Fig. 1. First visit panoramic view. No definitive bone necrosis except bone resorption was not observed on peri-implant area.

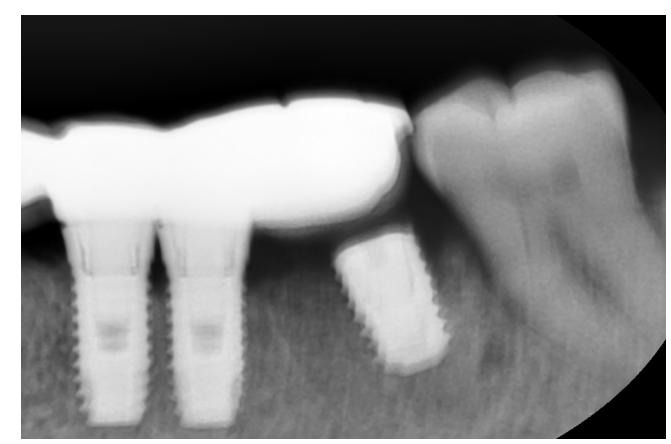

Fig. 2. First visit periapical view. Mild bone loss on mandibular left first molar implant area. 
was prescribed. If necessary, the removal of the implants was planned and considering the possibility of development or spread of MRONJ, it was decided to stop the antiresorptive agent in consultation with the medical doctor who prescribed it about its replacement or discontinuation. Afterwards, follow-up was planned but not made. Five months later, the patient revisited authors' clinic via the emergency room with chief complaint of continuous gingival swelling and pain of same area, new fistula formation on left submandibular area and pus discharge. Due to limitation of the mouth opening, detailed clinical examination and intraoral observation were not performed well, then for further evaluation, contrast enhanced computed tomography (CT) was conducted on the maxillofacial region. On CT view, pus cavity was seen on from mandibular inferior margin to retromandibular area (Fig. 3A and 3B), sequestrum formation with fracture line was seen around peri-implant area from mandibular left canine to mandibular left second molar (Fig. 3C, 3D and 3E). At the time of the emergency room visit, since the patient's hemoglobin level was very low at $3.7 \mathrm{~g} / \mathrm{dl}$, packed red blood cell transfusion was performed and it was improved to $6.4 \mathrm{~g} / \mathrm{dl}$. Subsequently, the patient was admitted to department of oral and maxillofacial surgery for active treatment considering the progress of the infection with the elderly. The low hemoglobin level was suspected to be due to iron deficiency anemia or gastrointestinal bleeding, then related examinations and treatments were conducted under consultation with internal medicine doctor. Management of dementia and delirium symptoms after the hospitalization was also performed.
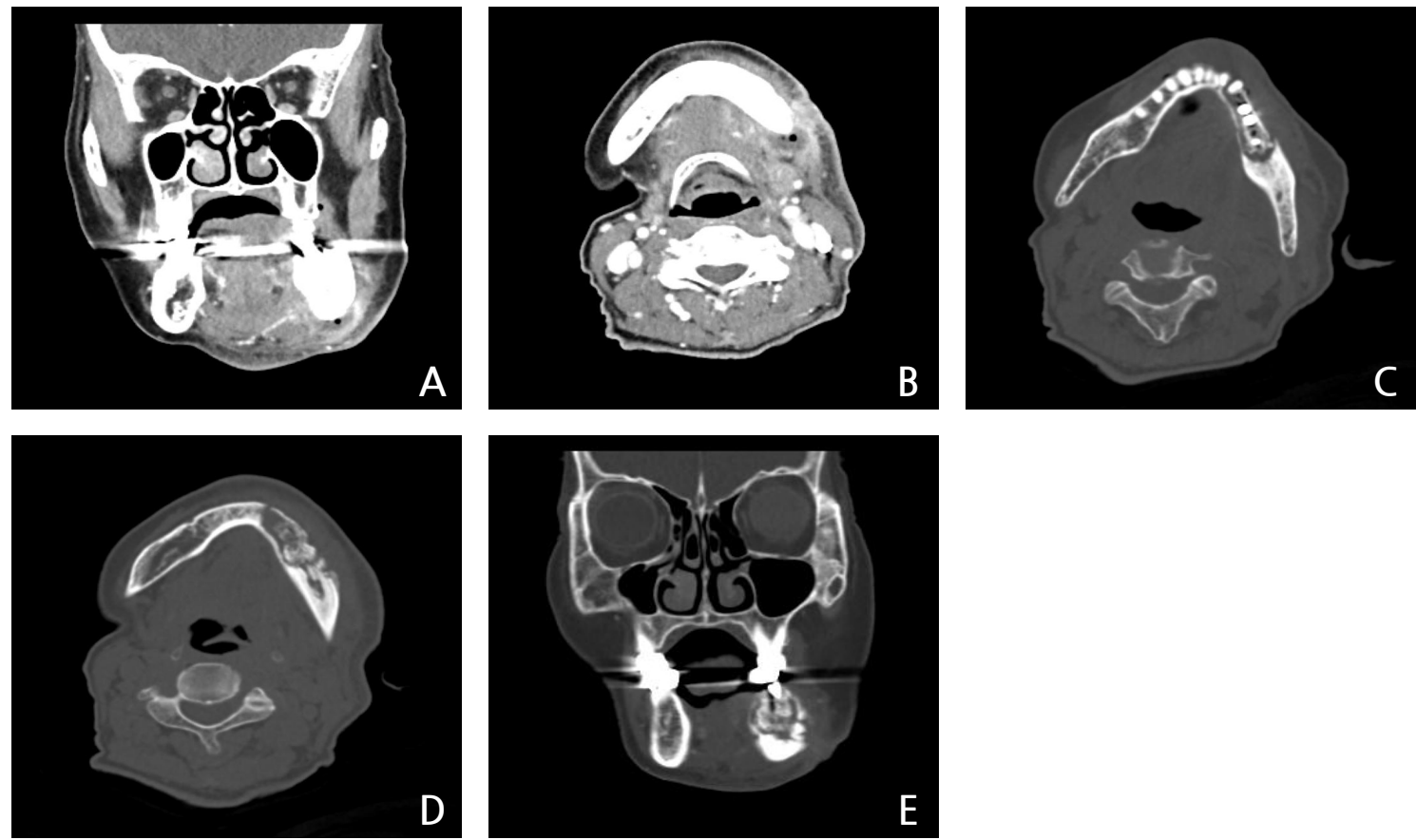

Fig. 3. Pre-operative CT view (A) Pus cavity was seen on inferior border of mandible. (B) Pus cavity was seen on Retromandibular area. $(C, D, E)$ Sequestrae and fracture line were seen on peri-implant area. 
Since general anesthesia for surgical treatment of osteonecrosis was impossible due to the patient's general condition, careful management and supportive care was performed and as the systemic condition improved, general anesthesia surgery was performed 8 days after hospitalization. The surgery was performed with extraction of \#33, 37, removal of \#34, 35, 36 implants and partial mandibulectomy of the affected area and reconstruction using a reconstruction metal plate. The intraoral findings of the patient could be observed in detail in the operation room, exposed necrotic bone with the implant fixture exposure and severe inflammation of surrounding soft tissue were seen (Fig. 4A). Compared to the time of admission, the submandibular fistula was consistently observed (Fig. 4B). Incision was approached from submandibular area, fracture line and sequestrum with penetrated buccal bone were seen at the operation site (Fig. 5A). The sequestrum was formed surrounding the implants, and the implant removal, tooth extraction were performed considering the affected area (Fig. 5B). Partial mandibulectomy was performed as planned, and the reconstruction metal plate was adapted in consideration of occlusion (Fig. 5C). Stable state of the reconstruction metal plate was confirmed on the radiograph immediately after the surgery (Fig. 6). No clinical and radiologic abnormalities were found 9 months after the surgery (Fig. 7A and $7 \mathrm{~B})$. On the panoramic view taken 15 months after the surgery, new bone was generated along the
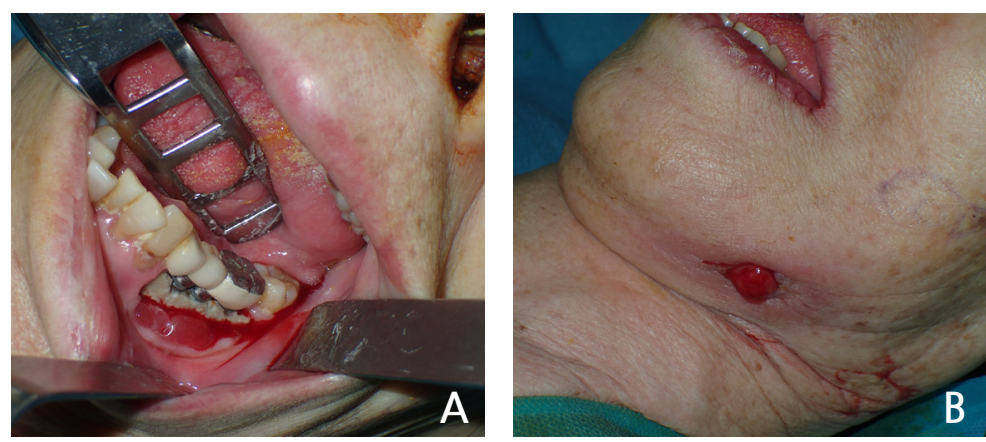

Fig. 4. Intra-operative clinical photo. (A) Exposure of necrotic bone surrounding implant fixture was seen. (B) Fistula formation was seen on left submandibular area.
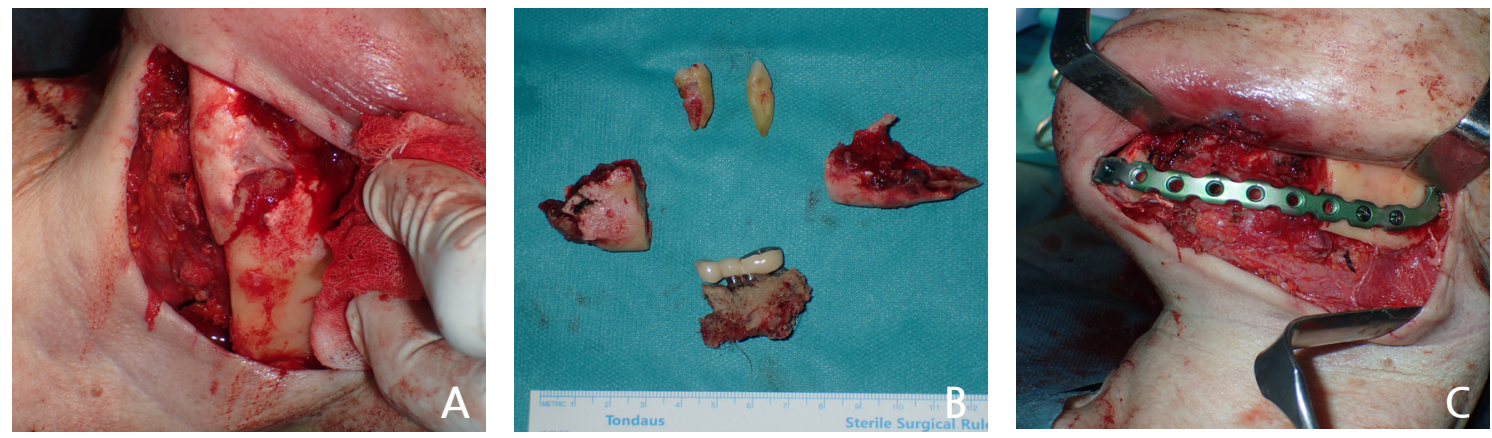

Fig. 5. Intra-operative clinical photo. (A) Fracture line and sequestrum with penetrated buccal bone were seen. (B) Sequestrum surrounding implant fixture, affected necrotic bone, extracted teeth. (C) Reconstruction plate was adapted. 
direction of the mandibular canal which is presumed to have been in the resected area (Fig. 8). Intravenous administration of antiresorptive agent (Denosumab) was given once 2 months after the surgery, but the operation site has been maintained well without any complication and recurrence.

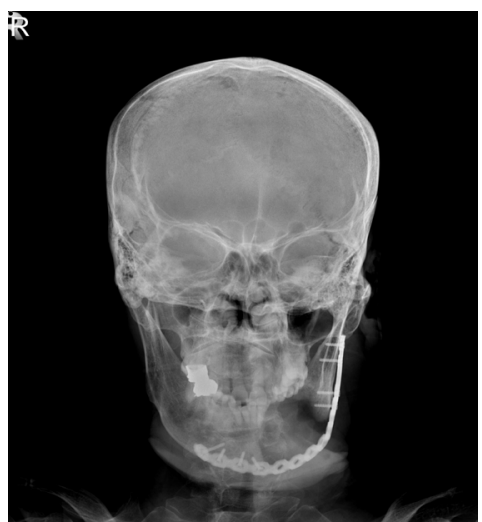

Fig. 6. Immediate Post-operative skull PA view shows stable operation site.
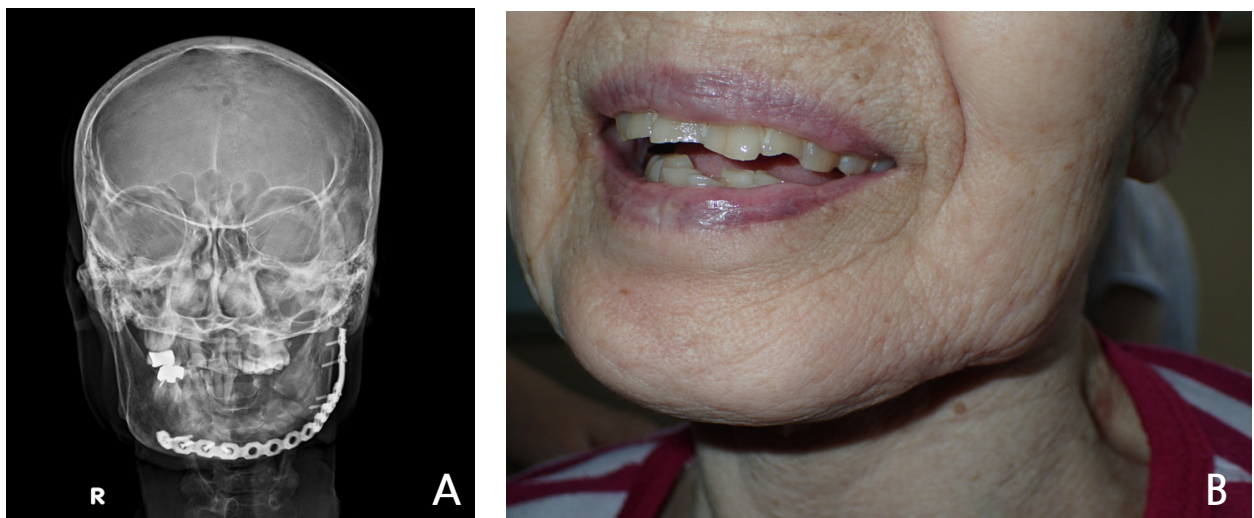

Fig. 7. 9 months after surgery. (A) Skull PA view shows well maintained operation site. (B) Facial photo shows no abnormal morphologic change.

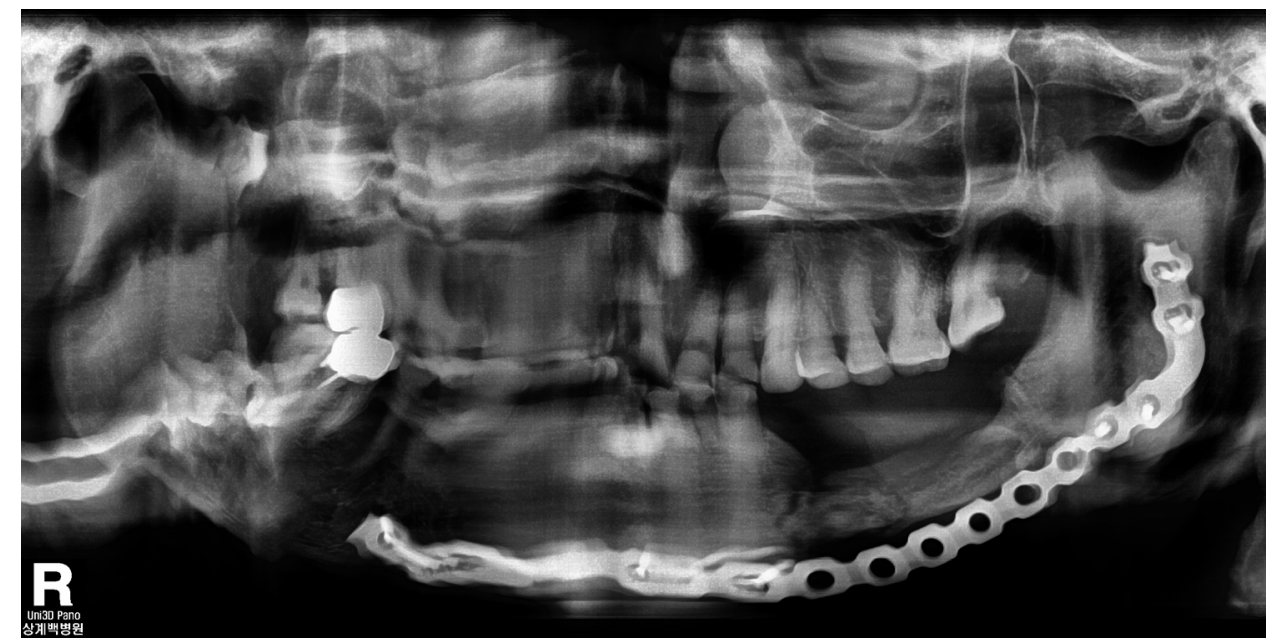

Fig. 8. 15 months after surgery. Panoramic view shows new bone was formed along the direction of mandibular canal which is believed to have been in the resected area. 


\section{Discussion}

MRONJ is a disease that oral and maxillofacial surgeons often encounter, but in fact, its prevalence is not high. In 2015, Dodson reported a literature review including studies published between 2008 and 2015, and reported the prevalence of MRONJ. ${ }^{8}$ According to the review, each studies showed that the risk of osteonecrosis ranged from $0 \%$ to $6.7 \%$ among patients with cancer exposed to intravenous antiresorptive or antiangiogenic agents. The best estimate of MRONJ among patients with cancer exposed to antiresorptive or antiangiogenic agents is in the low single digit percentages, $1 \%$ to $3 \%$. Also, it was reported that the risk of osteonecrosis among patients managed for osteoporosis using antiresorptive agents is about $0.1 \%$, ranged $0.004 \%$ to $0.2 \%$. Dodson described that the average general dentist may expect 1 new case of MRONJ for every 62 years in practice. However, despite the low prevalence, tooth extraction, dento-alveolar surgery and local infection can increase the incidence of MRONJ. Clinicians are often faced with situations where the possibility of development of MRONJ should be informed to patients, and many clinicians and patients want to know the figure of incidence rate of MRONJ. Dodson reported that osteonecrosis may occur in about $0.5 \%$ after tooth extraction in patients exposed to oral bisphosphonates to manage osteoporosis, but the risk of osteonecrosis after implant placement is unknown. Therefore, more reliable data and report should be accumulated and researched.

In 2017, Aljohani et al reported a systematic review about the effect of antiresorptive agents on the development of MRONJ in osteoporosis patients. 680 cases of MRONJ were analyzed in a total of 44 studies that were reviewed. As a result, the average age of patients with MRONJ was $69.7 \pm 5.2$ years, and it was found that it occurred to women at a rate of $93.5 \%$. Mandible was the most common site at a rate of $70.6 \%$. Alendronate was the most common antiresorptive agents at a rate of $72.6 \%$, oral administration was $86.7 \%$ of patients managed with bisphosphonates. The average duration of administration of oral or intravenous bisphosphonates was $51.9 \pm 18$ months. Tooth extraction was the most frequently preceding event at a rate of $48.5 \%$, and dento-alveolar surgery such as implant placement, implant removal, ridge augmentation is $21.1 \%$.

As described above, tooth extraction is the largest proportion as the cause of development of MRONJ, but considering the frequency of implant surgery performed in clinical practice, implant-related MRONJ should be concerned. Bedogni et al performed literature review about cases of BRONJ after implant surgery in patients taking oral bisphosphonates, and reported that the mean duration of bisphosphonates therapy was 5.5 years, most patients were given alendronate $70 \mathrm{mg}$ once a week. ${ }^{10}$ The patient in our case report also received alendronate at the same dose and regimen as the above report, but the duration of administration was 28 months, shorter than the average duration as the above report. Although some 
studies described that oral administration of bisphosphonates may increase the incidence of osteonecrosis after prolonged administration for more than 4 years, our case suggests that it cannot be an absolute criterion about decision of dento-alveolar surgery.

MRONJ related to implant can be classified into two types based on the trigger and onset, the one is case of MRONJ that developed after implant placement in patients who have been receiving antiresorptive or antiangiogenic agents, the other is, like our case, MRONJ that developed after administration of antiresorptive or antiangiogenic agents while the implant was already osseointegrated and functioning. In 2016, Giovannacci et al in their study defined osteonecrosis immediately after implant placement (from 2 to 10 months) as "implant surgery-triggered" MRONJ, and defined osteonecrosis distant from implant placement (from 1 to 15 years) as "implant presence-triggered" MRONJ." 8 of 9 "implant presence-triggered" MRONJ patients received intravenous bisphosphonates for malignant disease and only one patient received oral bisphosphonates, alendronate for osteoporosis. According to their study, although "implant presence-triggered" MRONJ has a lower incidence in patients who received oral bisphosphonates to treat osteoporosis than patients who received intravenous bisphosphonates to treat malignant diseases, but the possibility of development of MRONJ should not be excluded even in the case of oral bisphosphonates.

In 2020, Escobedo et al used the terms "implant presence-triggered osteonecrosis" (IPTO) and “implant surgery-triggered osteonecrosis" (ISTO). In their systematic review, 27 out of 114 patients with implant-related MRONJ in 9 studies were ISTO group, and 74 patients were IPTO group. ${ }^{12}$ As a result, it was reported that osteonecrosis associated with implants develops more frequently on implants that are already functioning than after implant placement surgery. In addition, it was reported that the average duration of administration in IPTO group (52.3 months) was longer than in ISTO group (41.4 months).

For the treatment of MRONJ, some clinicians prefer long-term antibiotic therapy, local rinse and conservative therapy, but many oral and maxillofacial surgeons prefer surgical protocols because an early and confined surgical removal of the necrotic bone limits the defect and avoids complications such as abscess or fistula formation or pathologic fractures. ${ }^{13,14}$ As our case, pathologic fractures may occur in MRONJ patients. Otto et al reported that 4 of 140 BRONJ patients $(2.9 \%)$ had pathologic fractures, ${ }^{15}$ Escobedoetal in their systematic review reported that 1 of 74 "implant presence-triggered osteonecrosis" patient (1.4\%) had pathologic fracture. ${ }^{12}$ In the case of pathologic fracture in MRONJ, rigid fixation using reconstruction metal plate may be more effective than fixation using miniplates, because of defect due to the removal of necrotic region. 
The spontaneous regeneration of bone in segmental mandibulectomy site is unexpected and unpredictable phenomenon, but this has been reported by several authors. Important suggested factors that can help spontaneous bone formation are masticatory stress under stable resected mandible, complete soft tissue protection and young age etc. ${ }^{16,17}$ This case showed new bone formation along the direction of mandibular canal which is presumed to have been in resected area (Fig. 8), and also it is notable that this type of bone regeneration has occurred in the elderly patient. More study will be needed on the factors affecting spontaneous bone regeneration in the resected jaw.

\section{Conclusion}

Osteoporosis is a common disease worldwide, and osteoporosis patients are predicted to increase each year. Bisphosphonate is one of the most widely used antiresorptive agents for the treatment of osteoporosis, then more studies of implant-related MRONJ caused by its use is necessary. This case report suggests the possibility of development of "implant presence-triggered" MRONJ. Clinicians should be aware that even if there were no problems at the time of implant placement, MRONJ may develop later around implants that are already present. Also, The possibility of development of MRONJ should not be excluded even in the case of short duration of oral bisphosphonates. Clinicians should inform the patient and medical doctor who plan the antiresorptive or antiangiogenic therapies that there is the possibility of development of MRONJ.

\section{References}

1. Hortobagyi GN, Theriault RL, Lipton A, Porter L, Blayney D, Sinoff C, et al. Long-term prevention of skeletal complications of metastatic breast cancer with pamidronate. Protocol 19 Aredia Breast Cancer Study Group. J Clin Oncol 1998;16:2038-44.

2. van Holten-Verzantvoort AT, Kroon HM, Bijvoet OL, Cleton FJ, Beex LV, Blijham G, et al. Palliative pamidronate treatment in patients with bone metastases from breast cancer. J Clin Oncol 1993;11: 491-8.

3. Marx RE. Pamidronate (Aredia) and zoledronate (Zometa) induced avascular necrosis of the jaws: a growing epidemic. J Oral Maxillofac Surg 2003;61:1115-7.

4. Ruggiero SL. Guidelines for the diagnosis of bisphosphonate-related osteonecrosis of the jaw (BRONJ). Clin Cases Miner Bone Metab 2007;4:37-42.

5. Ruggiero SL, Dodson TB, Fantasia J, Goodday R, Aghaloo T, Mehrotra B, et al. American association of oral and maxillofacial surgerons position paper on medication-related osteonecrosis of the jaw 2014 update. J Oral Maxillofac Surg 2014;72:1938-56.

6. Marx RE, Sawatari J, Fortin M, Broumand V. Bisphosphonate-induced exposed bone(osteonecrosis/ 
osteopetrosis) of the jaws: risk factors, recognition, prevention, and treatment. J Oral Maxillofac Surg 2005;63:1567-75.

7. Kwon TG, Lee CO, Park JW, Choi SY, Rijal G, Shin HI. Osteonecrosis associated with dental implants in patients undergoing bisphosphonate treatment. Clin Oral Implants Res 2014;25:632-40.

8. Dodson TB. The frequency of medication-related osteonecrosis of the jaw and its associated risk factors. Oral Maxillofac Surg Clin North Am 2015;27:509-16.

9. Aljohani S, Fliefel R, Ihbe J, Kühnisch J, Ehrenfeld M, Otto S. What is the effect of anti-resorptive drugs (ARDs) on the development of medication-related osteonecrosis of the jaw (MRONJ) in osteoporosis patients: a systematic review. J Craniomaxillofac Surg 2017;45:1493-1502.

10. Bedogni A, Bettini G, Totola A, Saia G, Nocin PF. Oral bisphosphonate-associated osteonecrosis of the jaw after implant surgery: a case report and literature review. J Oral Maxillofac Surg 2010;68:1662-6.

11. Giovannacci I, Meleti M, Manfredi M, Mortellaro C, Lucchina GA, Bonanini M, et al. Medicationrelated osteonecrosis of the jaw around dental implants: implant surgery-triggered or implant presence-triggered osteonecrosis? J Craniofac Surg 2016;27:697-701.

12. Escobedo MF, Cobo JL, Junquera S, Milla J, Olay S, Junquera LM. Medication-related osteonecrosis of the jaw. Implant presence-triggered osteonecrosis: case series and literature review. J Stomatol Oral Maxillofac Surg 2020;121:40-8.

13. Stanton DC, Balasanian E. Outcome of surgical management of bisphosphonate-related osteonecrosis of the jaws: review of 33 surgical cases. J Oral Maxillofac Surg 2009;67:943-50.

14. Markose G, Mackenzie FR, Currie WJR, Hislop WS. Bisphosphonate osteonecrosis: a protocol for surgical management. Br J Oral Maxillofac Surg 2009;47:294-7.

15. Otto S, Pautke C, Hafner S, Hesse R, Reichardt LF, Mast G, et al. Pathologic fractures in bisphosphonate-related osteonecrosis of the jaw-review of the literature and review of our own cases. Craniomaxillofac Trauma Reconstr 2013;6:147-54.

16. Elbeshir EI. Spontaneous regeneration of the mandibular bone following hemimandibulectomy. $\mathrm{Br}$ J Oral Maxillofac Surg 1990;28:128-30.

17. Nwoku AL. Unusually rapid bone regeneration following mandibular resection. J Maxillofac Surg 1980;8:309-15. 